Volume 9, No.3, May - June 2020

International Journal of Advanced Trends in Computer Science and Engineering

Available Online at http://www.warse.org/IJATCSE/static/pdf/file/ijatcse232932020.pdf

https://doi.org/10.30534/ijatcse/2020/232932020

\title{
The Fast Integration of a Rotated Haar-Like Feature for Face Detection
}

\author{
Mohamed OUALLA ${ }^{1}$, Khalid OUNACHAD ${ }^{2}$,AbdelhalimHNINI ${ }^{3}$,Abdelalim SADIQ ${ }^{4}$ \\ ${ }^{1}$ Moulay Ismail University, FST, Errachidia, Morocco, mohamedoualla76@gmail.com \\ ${ }^{2}$ Ibn Toufail University, FS, Kénitra, Morocco, ounachad@gmail.com \\ ${ }^{1}$ Hassan $1^{\text {st }}$ University, ENSA,Berrechid. Morocco, hninihalim@gmail.com \\ Ibn Toufail University FS, Kénitra, Morocco,a.sadiq@uit.ac.ma
}

\begin{abstract}
This paper addresses the problem of detecting rotated human faces in images. We propose a new face detection approach based on a novel extension of rotated Haar-like features, and a method to calculate their values based on the Bresenham algorithm of drawing a segment. The extension of the basic set of features, proposed by Viola and Jones, is done by the generation of a set of rotated Haar-likefeatures, by any angle, in an image. For the feature's integration process, we calculate the value of a rotated feature using the integral image technique, proposed by viola and jones. The main objective of this article is to propose a method to calculate the integral image of a rotated feature; we introduce the concept of key points that forms the four segments of a rotated rectangle. Our method focuses on three essential tasks; the first is to determine the rule for drawing a segment (SDR), the second is to identify all the key points of the rectangle $r$, and the third is to calculate the integral image. The speed of this method depends on the size and angle of rotation of the rectangle. Experimental results obtained using images from the MIT-CMU dataset are promising in terms of detection rate and the false alarm rate in comparison with other competing algorithms.
\end{abstract}

Key words; Haar-Like features, Integral image, Face detection, Object detection, Viola \& Jones algorithm, AdaBoost.

\section{INTRODUCTION}

Face detection has become a hot area of research in recent years. Face detection is the key technology and necessary prerequisite for face recognition, expression recognition, digital video processing and video detection[19][20]. Adaboost learning algorithm, proposed by [1], and adapted by Viola and Jones for object detection [2][3], is one of the most widely used algorithms in detection. This algorithm, based on Haar-like features (Figure. 2), achieves a high detection rate and we use it widely in face and pedestrian detection [4][5][6].

Haar-like feature classifiers trained by Adaboost algorithm are often incapable of finding rotated objects (Figure. 1). Many experts have proposed several solutions to fill this problem; Viola and Jones [2][3][4] used rotated positive examples during training, but this approach may give some inaccurate classifier. Another process adopted by several researchers [5 ][7] [9] [10] consists of training several

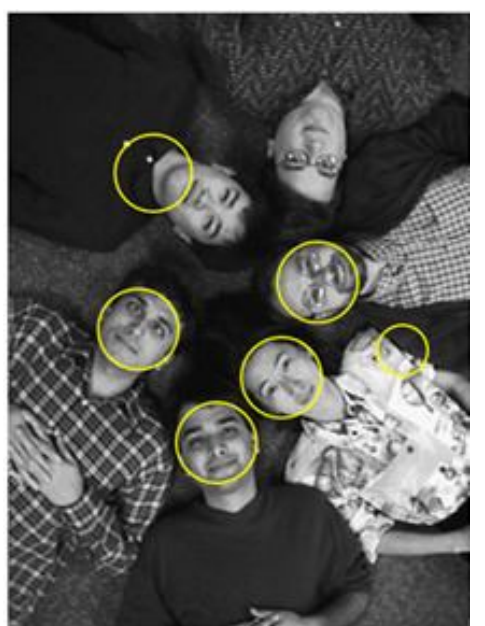

Figure 1: Face detection with the latest method [12]; The algorithm is unable to detect a few rotated faces.

classifiers which specialize in certain angle intervals, this approach provides classifiers with appropriate accuracy and efficiency, but it makes the training computationally more expensive. In [7][8]the image is physically or virtually rotated until the edges of the Haar-Like feature are aligned vertically or horizontally, this approach makes the detection computationally more expensive and also a loss of information when a set of pixels will be outside the region of interest.

In [12], we gave a solution allowing, firstly, to define, dynamically, a set of rotated Haar-Like Features by more than 50 angles formally expressed by $\operatorname{arctang}(A / B)$ such that $A$ and $B$ are two integers. And secondly, to calculate, approximately, the rotated integral image of these rectangles to preserve the two advantages (speed and simplicity) for which Viola and Jones invented this technique for the first time. This method has shown a disadvantage, a little annoying, which consists of a false alarm rate, which is relatively high.

In this paper, we propose a method that calculates the real Integral Image of a rotated rectangle based on the set of pixels, called key-points, forming each of its four segments. Indeed, we determine these points by adapting the algorithm for drawing a segment [13]. 
We organize this paper as follows: Section II presents some work done in the literature. In section III we will propose our technique concerning the quickly integration of a rotated rectangle. Section IV explores the results obtained

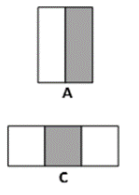

(a)

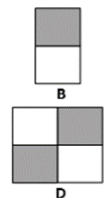

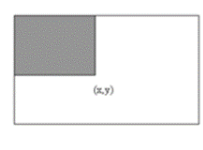

(b)

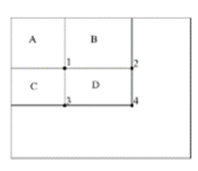

(c)
Figure 2: (a) Haar-Like basic features, used by ViolaJones in [2]. (b) Integral Image (c) Calculation of the sum of the rectangle D with the Integral Image.

by our two experiments carried out for the case of face detection. In section $\mathrm{V}$, we will give a summary of our work, some conclusions, and perspectives.

\section{RELATED WORK}

The technique of the Integral Image, strongly associated with the famous algorithm of Viola and Jones [2], is a technique that goes back to the year 1984 when Crow [16] introduced it, for the first time, in computer graphics. Its use in computer vision began in 2001 when Viola and Jones used it to calculate the integral of the rectangular filters called HaarLike features, shown in Figure.2 (a), considered as one of the pillars of their real-time face detection algorithm. Therefore, most researchers have widely used and developed this technique to solve the problems related to the detection of objects, including faces detection[2][7], pedestrian detection [4][6], etc.

Let $i$ be an image of dimension $M \times N, i(x, y)$ is the intensity of the pixel $(x, y)$ of the image $i$. The Integral Image is another image $i i$ such that for each pixel $(x, y)$ : (see Figure. 2(b)).

$$
i i(x, y)=\sum_{\substack{x^{\prime} \leq x \\ y^{\prime} \leq y}} i\left(x^{\prime}, y^{\prime}\right)
$$

Therefore, the integration of each axis-aligned (parallel to the axes of the image coordinate system) rectangle $R=[(x, y), w, h]$ is calculated only in four references: (see Figure. 2(c))

$$
\begin{gathered}
i i(R)=i i(x+w, y+h)-i i(x, y+h) \\
-i i(x+w, y)+i i(x, y)
\end{gathered}
$$

As $(x, y)$ is the upper left corner of $R, w$ and $h$ represent, respectively, its width and height. In the rest of this article, this type of rectangle is called a normal rectangle. This technique was then the subject of several improvements, in order to detect rotated objects in an image. Indeed, several works have chosen to feed the original set of HaarLike features, initially proposed by Viola and Jones (Figure.2 (a)), by others which are rotated by a variety of angles and offering an ease of calculating their integration. In particular, Lienhart et al. [5] who introduced a $45^{\circ}$ tilted integral image. Then, Barczak et al. [7] and Du et al. [8] retained the same technique of Viola and Jones to incorporate rotated featuresby $26.57^{\circ}$ and $63.5^{\circ}$. Then Barczak and Mossom [9] tried to generalize

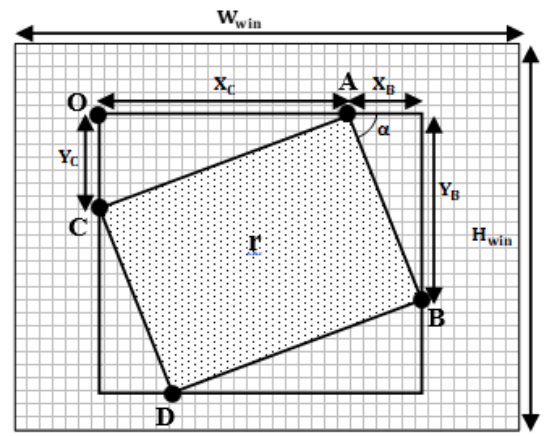

Figure 3: Representation of a rotated rectangle.

this technique for angles having a tangent in the form of a rational number $1 / n$ or $n / 1$. Another work is that of Ramirez et al. [10], who introduced the asymmetric Haar Features. Pham et al. [14] have developed a technique called Polygonal integration to divide a polygon into a set of axis-aligned rectangles (normal rectangles), then determine its integration according to those of theserectangles. Doretto et al. [15] gave an extension of formula 1 to compute the integral of a domain $D \subset \mathbb{R}^{n}$, which consists of a finite unification of axis-aligned rectangles.

\section{FAST INTEGRATION OF THE ROTATED HAAR-LIKE}

\subsection{Rotated Haar-Like features}

A rotated Haar-Like feature is a rectangle rotated by a given angle. The rotated Haar-like features are based on the normal ones presented in [2]. In a scan window named Win - with $H_{\text {win }}$ as height and $W_{\text {win }}$ as width - a rotated rectangle is defined by its vertex $A\left(x_{A}, y_{A}\right)$, its rotation angle $\alpha$ and its rectangle encapsulating $R\left[o\left(x_{A}-\right.\right.$ $\left.\left.x_{C}, y_{A}\right), w=X_{C}+X_{B}, h=Y_{B}+Y_{C}\right]$ as shown in Figure. 3.

Formally, a rotated rectangle is defined by a six-element vector as shown by the following formula:

$$
\begin{aligned}
& r_{A}^{t}=\left(A, X_{B}, Y_{B}, X_{C}, Y_{C}, \alpha\right) \in \operatorname{Win} \times\left(\mathbb{N}^{*}\right)^{4} \times \mathbb{R}^{*} \\
& \text { such as } n_{\alpha}=\frac{Y_{B}}{X_{B}}=\frac{X_{C}}{Y_{C}} \\
& \text { and } \alpha=\arctan \left(n_{\alpha}\right) / n_{\alpha} \in \mathbb{Q}^{*} \\
& \text { and } Y_{B}+Y_{C}+y_{A}<H_{\text {win }}, \quad X_{B}+x_{A}<W_{\text {win }}
\end{aligned}
$$

The set of rotated rectangles $\mathcal{R}$ is divided into two categories $\mathcal{R}_{a}$ and $\mathcal{R}_{b}$. These two categories are defined by formulas 4 and 5 .

$$
\begin{gathered}
\mathcal{R}_{a}=\left\{r \in \mathcal { R } | ( y _ { B } \geq y _ { c } \operatorname { E T } x _ { A } \geq x _ { D } ) \text { OU } \left(y_{B}\right.\right. \\
\left.\left.\leq y_{c} E T x_{A} \leq x_{D}\right)\right\} \\
\mathcal{R}_{b}=\left\{r \in \mathcal { R } | ( y _ { B } > y _ { c } E T x _ { A } < x _ { D } ) \text { OU } \left(y_{B}\right.\right. \\
\left.\left.<y_{c} E T x_{A}>x_{D}\right)\right\}
\end{gathered}
$$

knowing that $B, C$ and $D$ are other vertices of the rotated rectangle $r$, as shown in Figure.3. 

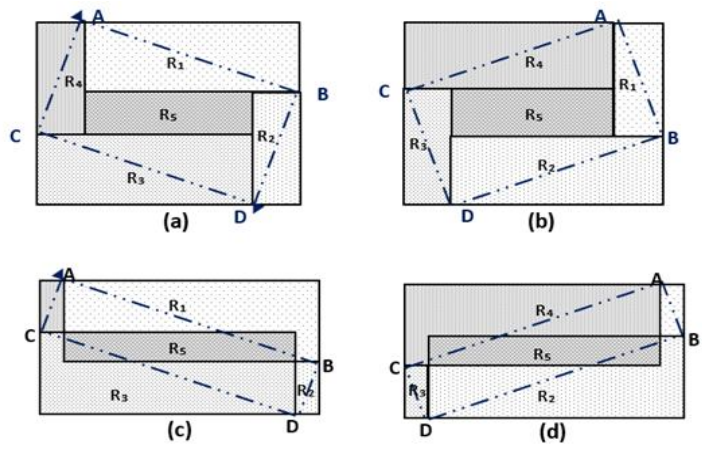

Figure 4: Representation of a rotated rectangle of the category $\mathcal{R}_{a}$ by (a) and (b) and $\mathcal{R}_{b}$ by (c) and (d).

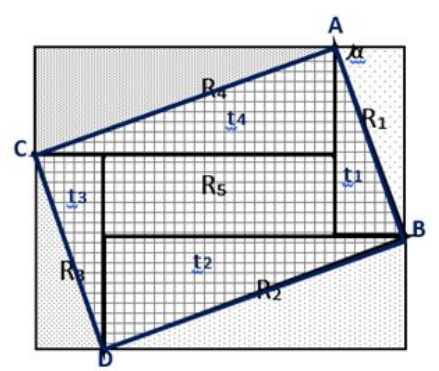

Figure 5: A rotated rectangle divided into triangles.

\subsection{The integration of a rotated rectangle}

The principle of our method consists in dividing the rectangle that encapsulates $r$ into five normal rectangles called $R_{\mathrm{i}}$, as shown in Figure. 4 and 5. The result of the intersection of $r$ with the rectangles $R_{\mathrm{i}}$ is the right triangle called $t_{i}$. We illustrated this definition in Figure. 5. The following equation gives the identification of these triangles: $t_{i}=r \cap R_{i}$ for $\quad i \in\{1,2,3,4\}$.

Knowing that the integral of a rotated rectangle is:

$$
I(r)=\iint_{(x, y) \in r} i(x, y)
$$

such that $i(x, y)$ is the intensity of the pixel $(x, y)$ belonging to the rectangle $r$. The exploitation of the integral image technique proposed by Viola and Jones [2] leads us to reformulate equation 6 in this form:

$$
I(r)=\sum_{i=1}^{4} \operatorname{In}\left(t_{i}\right)+S \times I\left(R_{5}\right) /\left\{\begin{array}{l}
S=+1 \text { ifr } \in \mathcal{R}_{a} \\
S=-1 \text { ifr } \in \mathcal{R}_{b}
\end{array}\right.
$$

such that: $I\left(R_{5}\right)$ is the Integral Image of $R_{5}$ rectangle calculated according to the equation $2 . \operatorname{In}\left(t_{i}\right)$ is the integration of the triangle $t_{i}$.

Indeed, we can divide a triangle $t$ into several normal rectangles $R_{\mathrm{i}}$ (axis-aligned rectangles) (Figure. 6). The two vertices $\left(M_{i}, N_{i}\right)$ of each rectangle $R_{\mathrm{i}}$ crossed by the hypotenuse of the triangle $t$ are named key points. The set of these key points, $\mathcal{P C}$, are found by applying the Segment Drawing Rule (SDR) [13]. In fact, $\mathcal{P C}=\mathcal{M} \cup$
$\mathcal{N}$ such that:

$$
\begin{gathered}
\mathcal{M}=\mathcal{M}_{t_{1}} \cup \mathcal{M}_{t_{2}} \cup \mathcal{M}_{t_{3}} \cup \mathcal{M}_{t_{4}} \\
\mathcal{N}=\mathcal{N}_{t_{1}} \cup \mathcal{N}_{t_{2}} \cup \mathcal{N}_{t_{3}} \cup \mathcal{N}_{t_{4}}
\end{gathered}
$$

Knowing that $\mathcal{M}_{t_{i}}$ and $\mathcal{N}_{t_{i}}$ are, respectively, the set of key points $M_{i}$ and $N_{i}$ of the triangle $t_{i}$.

Mathematically speaking, let $f: \mathcal{R} \rightarrow \mathcal{P C}^{n}$ be a function that determines the vector $v_{p}=\left(P_{0}, \ldots, P_{n}\right)$ of the key points, for each rectangle $r$ of $R$, therefore: $f(r)=v_{p}=$ $\left(P_{0}, \ldots, P_{n}\right)$. And $g: \mathcal{P C} \mathcal{C}^{n} \rightarrow \mathbb{R}$ a function that computes the Integral Image from a vector of points. In other words, $g\left(v_{p}\right)=I(r)$. Indeed, we define the function I as a function composed of $f$ and $g: I(r)=f o g(r)$.

Therefore, the method proposed in this article, to calculate the Integral Image of a rectangle, is based on three essential tasks:

- Determine the rule for drawing a segment (SDR);

- Determine all the key points $\left(M_{i}, N_{i}\right)$ of the rectangle $r$;

- Calculate the integral image of $r$ according to its key points.

\subsection{Triangle integration}

The major problem of the computation part of the integral of a right triangle $t$ is that each slope of its hypotenuse presents different difficulties. Is very difficult to integrate a line with an irrational slope because at the level of each pixel intersected by the line, the partition model is always different. For our case, the slope of the hypotenuse of the triangle $t$ is rational. Specifically, a line with a rational slope produces a finite number of pixel partition models.

Consider, for example, a positive slope $n$ of a line $L$, when this line crosses a set of pixels, the model of partitions of the pixels is repeated at each $n$ pixels intersected by the line $L$ [14]. This repetition is the key of our solution proposed in this article. More generally, let $d=n / m(n, m \in Z$ and $m \geq 0)$ be a rational slope, the integer vector $v_{d}=(m, n) / \operatorname{gcd}(|n|,|m|)$ represents a $2 \mathrm{D}$ interval such as the partition model is periodic. Otherwise, the partition model at any pixel $(x, y)$ is the same at $(x, y)+v_{d}$. To determine the pixels traversed by a line $L$, we used the Bresenham algorithm [13], which makes it possible to define the Segment Drawing Rule (SDR).

A. Segment Drawing Rule (SDR):

Based on the Bresenham algorithm [13], which allows determining all the pixels forming a segment, we have defined the rule allowing to go through all the pixels forming the hypotenuse of a triangle $t$ (Figure. 6 (a)). This rule $R_{S D R}$ is a vector formally defined as follows: $R_{S D R}=\left(n_{1}, n_{2}, \ldots, n_{e}\right)$ such that $e$ represents the number of floors which is equal to $\min \left(Y^{\prime}, X^{\prime}\right)$ and $n_{i}$ that of pixels for the $i^{\text {th }}$ floor; Figure. 6 (b) gives an example of these values. A 


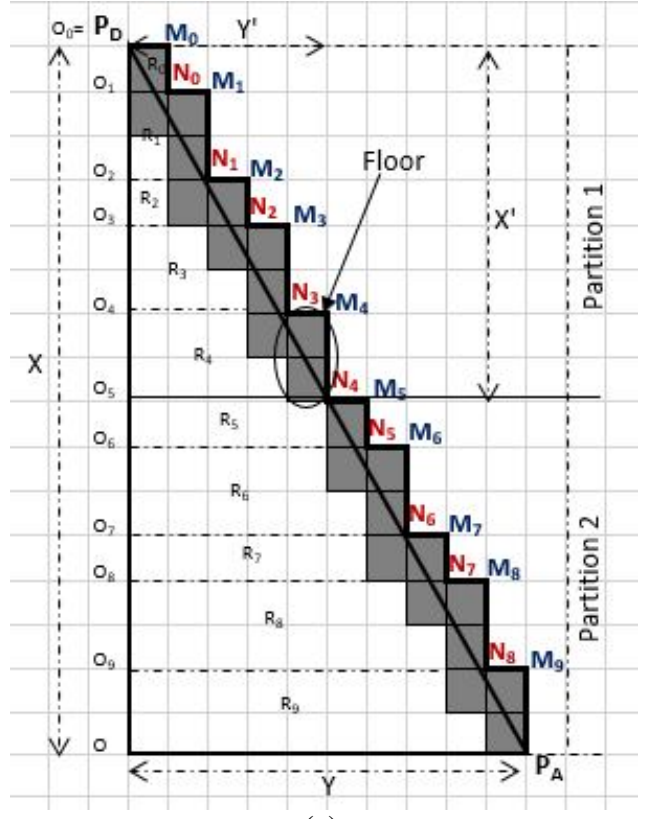

(a)

\begin{tabular}{|c|c|}
\hline$(\boldsymbol{X}, \boldsymbol{Y})$ & $(16,10)$ \\
\hline $\boldsymbol{N}=\operatorname{gcd}(|\boldsymbol{X}|,|\boldsymbol{Y}|)$ & 2 \\
\hline$\left(\boldsymbol{X}^{\prime}, \boldsymbol{Y}^{\prime}\right)=(\boldsymbol{X}, \boldsymbol{Y}) / \boldsymbol{N}$ & $(8,5)$ \\
\hline $\boldsymbol{e}=\boldsymbol{\operatorname { m i n }}\left(\boldsymbol{X}^{\prime}, \boldsymbol{Y}^{\prime}\right)$ & 5 \\
\hline $\boldsymbol{R}_{S D R}=\left(\boldsymbol{n}_{1}, \boldsymbol{n}_{2}, \ldots, \boldsymbol{n}_{\boldsymbol{e}}\right)$ & $R_{S D R}=(2,3,2,3,2)$ \\
\hline $\boldsymbol{k}$ & 10 \\
\hline $\boldsymbol{d}$ & vertical -right \\
\hline
\end{tabular}

Figure 6:(a) Rule for drawing a segment, (b) Example of values for $X=16, Y=10$.

floor is a set of $n_{\mathrm{i}}$ pixels aligned either vertically (if $\alpha<$ $45^{\circ}$ ) or horizontally (if $\alpha>45^{\circ}$ ). The transition from one floor to another is done by moving a pixel to the right for sides $[A B]$ and $[C D]$ and left for $[A C]$ and $[B D]$. A particular case is that of $\alpha=45^{\circ}$, the number of pixels for each floor is always equal to 1 , and the displacement can be done in both directions.

Indeed, the Segment Drawing Rule (SDR) is the result of the function $s: \mathbb{S} \rightarrow \mathbb{N}^{e}$ which takes in parameter a segment $S$ and returns a vector $R_{S D R}=\left(n_{1}, n_{2}, \ldots, n_{e}\right) . S$ is the set of segments of a polygon; for our case, we are talking about a rectangle with four segments. Eachsegment is formally defined as follows: $S=$ $\left(P_{D}, P_{A}, X^{\prime}, Y^{\prime}, N, k, d\right)$, such that:

- $P_{D}$ and $P_{A}$ are, respectively, the starting point and the arrival point of the segment $S$;

- $\quad N=\operatorname{gcd}(|X|,|Y|)$ is the number of partitions or repetitions of $R_{S D R}$;

$$
\left(X^{\prime}, Y^{\prime}\right)=(X, Y) / N
$$

- $k=\min (X, Y)=\min \left(X^{\prime}, Y^{\prime}\right) * N=e * N$ : the number of floors composing the hypotenuse of the triangle;

- $d$ : Represents the direction of the path to follow, to browse all the pixels:

if $\alpha<45^{\circ}$ then $d \in$ \{vertical-right,vertical-left $\}$

if $\alpha>45^{\circ}$ then $d \in$ \{horizontal-right,horizontalleft

Algorithm 1 shows the definition of the segment drawing rule $(\mathrm{SDR})$ for both segments $[A B]$ and $[C D]$ with a direction $d=$ Vertical - Right.

The authors of [14] have shown that the number of pixels, denoted $\mathrm{np}$, traversed by the hypotenuse of the right triangle $t$ for each partition is $n p=\left|X^{\prime}\right|+\left|Y^{\prime}\right|-1$, indeed, we easily deduce that the total number of pixels traversed by the hypotenuse of $t$ is: $n p \times N$. Therefore, to determine the SDR, algorithm 1 traverses $(n p+e) \times$ $N$ pixels.

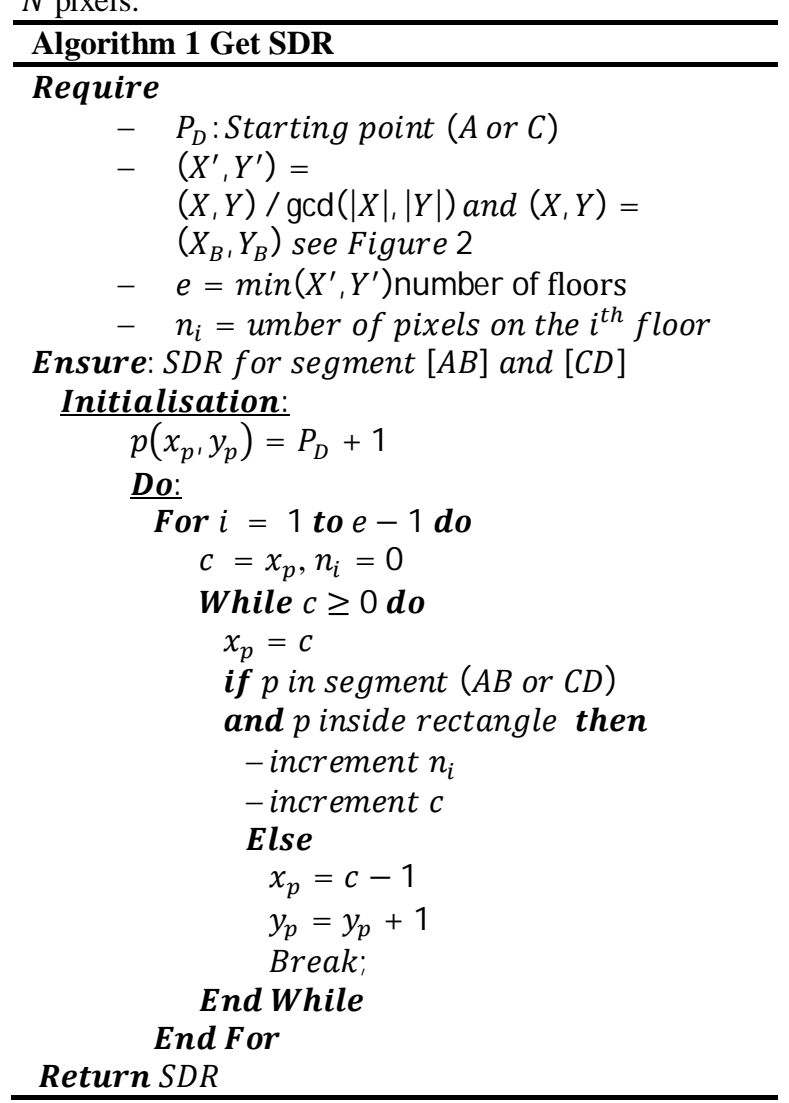




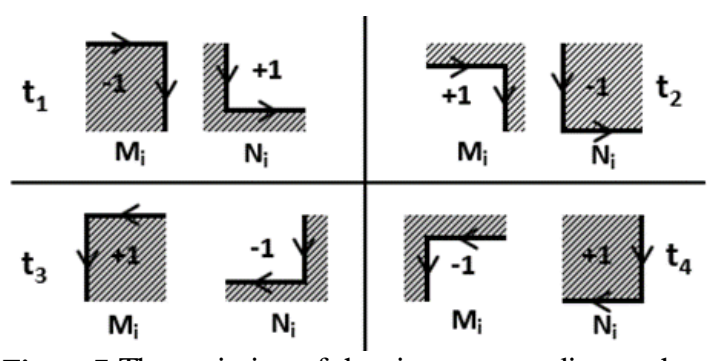

Figure 7: The variation of the sign $\rho_{i}$ according to the type of the triangle and the key point $(M$ or $N)$

B. Integral image of a right triangle:

As mentioned above, the principle of our method is based on the division of a triangle into k normal rectangles $R_{i}$. A normal rectangle $R_{i}$ is defined by the following quadruple: (see Figure.6 (a)) $R_{i}=\left[O_{i} M_{i} N_{i} O_{i+1}\right]$ fort $_{1}$, such us :

- $t_{1}$ is the triangle with the hypotenuse as segment $[A B]$, shown in Figure5;

- $\quad k$ : Number of floors;

- $i=0,1, \ldots, k-1$;

- $N_{k-1}=P_{A}$ and $O_{0}=P_{D}$;

- $O_{k}=O$.

Therefore, the Integral Image of a triangle $t$ will be the sum of those of all rectangles $R_{i}$, formally:

$$
\operatorname{In}(t)=\sum_{0}^{k-1} \operatorname{Im}\left(R_{i}\right) / k \text { : number of rectangles } R_{i} .
$$

Indeed, the Integral Image of a normal rectangle $R_{i}$, $\operatorname{Im}\left(R_{\boldsymbol{i}}\right)$, according to formula 2 is:

$$
\operatorname{Im}\left(\boldsymbol{R}_{\boldsymbol{i}}\right)=i i\left(N_{i}\right)-i i\left(O_{i+1}\right)-i i\left(M_{i}\right)+i i\left(O_{i}\right)
$$

Such as the function $i$ represents the Integral Image of a given pixel.

Therefore, from equation 8 and 9, we deduce the formula to calculate the Integral Image of a triangle $t$ (case of triangle $\left.t_{1}\right)$ :

$$
\begin{aligned}
\operatorname{In}(t)=\sum_{0}^{k-1} \operatorname{Im}\left(\boldsymbol{R}_{\boldsymbol{i}}\right) & \\
& =i i\left(P_{D}\right)+i i\left(P_{A}\right)-i i(O) \\
& +\sum_{0}^{k-2} i i\left(N_{i}\right)-\sum_{0}^{k-1} i i\left(M_{i}\right)
\end{aligned}
$$

Knowing that for $t_{1}: P_{D}=A e t P_{A}=B$.

According to this principle, we deduce the formulas for calculating the integral image of all types of triangles of the rotated rectangle $r$ : (Figure. 5)

$$
\begin{aligned}
& \operatorname{In}\left(t_{1}\right)=i i(A)+i i(B)-i i(O)-\sum_{0}^{k-1} i i\left(M_{i}\right) \\
& +\sum_{0}^{k-2} i i\left(N_{i}\right) \\
& \operatorname{In}\left(t_{2}\right)=i i(O)-\sum_{0}^{k-1} i i\left(M_{i}\right)+\sum_{0}^{k-2} i i\left(N_{i}\right) \\
& \operatorname{In}\left(t_{3}\right)=-i i(O)+\sum_{0}^{k-1} i i\left(M_{i}\right)-\sum_{0}^{k-2} i i\left(N_{i}\right) \\
& \operatorname{In}\left(t_{4}\right)=-i i(A)-i i(C)+i i(O)+\sum_{0}^{k-1} i i\left(M_{i}\right) \\
& -\sum_{0}^{k-2} i i\left(N_{i}\right)
\end{aligned}
$$

\subsection{Rotated Rectangle Integration}

In the end and according to equation 7 , formula 15 shows the Integral Image of a rotated rectangle. That of the rectangle of the medium $R_{5}$ is easily found via the equation 2 .

$$
\begin{aligned}
I(r)=i i(B)-i i(C)) & -\sum_{0}^{k-1} i i\left(M_{1 i}\right) \\
& +\sum_{0}^{k-2} i i\left(N_{1 i}\right)-\sum_{0}^{l-1} i i\left(M_{2 i}\right) \\
& +\sum_{0}^{l-2} i i\left(N_{2 i}\right)+\sum_{0}^{k-1} i i\left(M_{3 i}\right) \\
& -\sum_{0}^{k-2} i i\left(N_{3 i}\right)+\sum_{0}^{l-1} i i\left(M_{4 i}\right) \\
& -\sum_{0}^{l-2} i i\left(N_{4 i}\right)
\end{aligned}
$$

Knowing that $k$ is the number of floors of the hypotenuses of triangles $t_{1}$ and $t_{2}$ and $l$ that of triangles $t_{2}$ and $t_{4}$. So, the total number of key points $P_{i}$ of a rotated rectangle $r$, adding the two points $B$ and $C$, is $n=4(k+l)-2$.

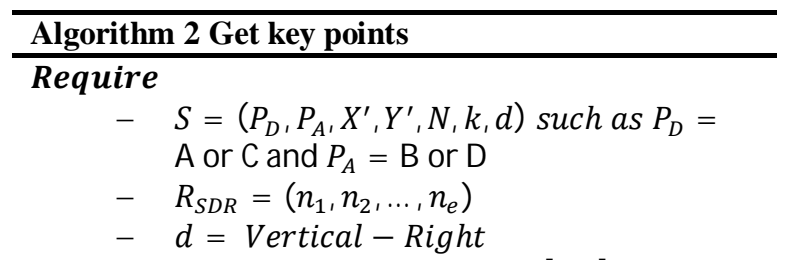

Ensure: Key points for segment $[A B]$ and $[C D]$ Initialisation: 
Do:

$$
M_{0}\left(x_{M_{0}}, y_{M_{0}}\right)=\left\{\begin{array}{c}
x_{M_{0}}=x_{P_{D}}+1 \\
y_{M_{0}}=y_{P_{D}}
\end{array}\right.
$$

$$
\begin{gathered}
\text { For } i=1 \text { to } k-1 \text { do } \\
x_{M_{i}}=x_{M_{i-1}}+1 \\
c=i \text { mod } e \\
\text { if } c<>0 \text { then } \\
y_{M_{i}}=y_{M_{i-1}}+n_{c-1}-1 \\
\text { Else } \\
y_{M_{i}}=y_{M_{i-1}}+n_{e-1} \\
x_{N_{i-1}}=x_{M_{i-1}} \\
y_{N_{i-1}}=y_{M_{i}} \\
\text { End For }
\end{gathered}
$$

Return key points

In general, the Integral Image of a rotated rectangle $r$ is:

$$
I(r)=\sum_{0}^{n-1} \rho_{i} * i i\left(P_{i}\right) / \rho_{i} \in\{+1,-1\}
$$

Figure.7 shows the variation of the sign $\rho_{i}$ according to the type of the triangle and the key point $(+1$, for $B$, and -1 for $C$ )

Algorithm 2 illustrates the process proposed to find these points for the two segments $[A B]$ and $[C D]$ with the direction of the path $d=$ Vertical - Right .

Indeed, to compute the Integral Image of a rotated rectangle, we need $4(k+1)-2$ access to the memory, which means that the associated algorithm has a linear complexity $\mathrm{O}(\mathrm{n})$. In practice, the worst-case complexity varies between 40 and 74 operations for only $12 \%$ of cases.

The complexity in the best case varies between 6 and 20 operations for more than $45 \%$ of cases, and 23 on average for all cases.

Such as $\rho_{i}$ is the parity corresponding to the $\operatorname{pixel} P_{i}$.

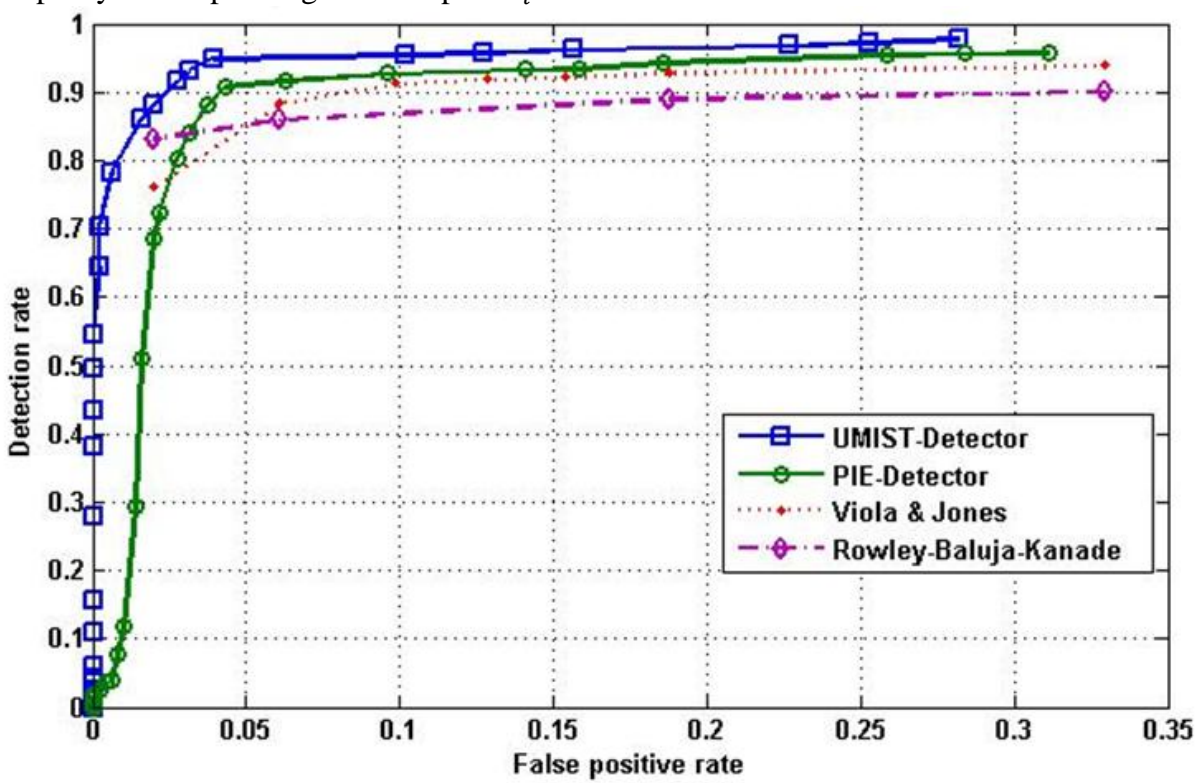

Figure 8: The ROC curve of UMIST-Detector, PIE-Detector, and other detectors using the MIT-CMU test base.

\section{EXPERIENCES}

During the learning phase, we adopted the use of two of the most known and most available databases in our field, which are: UMIST Face Database [17] and CMU-PIE Face Database [18]. The base of faces UMIST consists of 564 images - cut and trimmed - of 20 people (mixed race, kind, and appearance) [17]. In this database we represent each person's frontal profile by a multitude of images illustrating a range of poses from different angles; their number is about 19 up to 36 for each person. These images are sampled, in our experience, up to a resolution of $20 \times 20$. Their number is 6900 images of faces. The CMU-PIE database contains 41,368 images obtained from 68 people. These images were taken in the CMU 3D room using a set of 13 high-quality color cameras synchronized with 21 flashes. The resulting RGB color images are 640 $\times 486$ in size. These images are sampled, in our experiment, up to a resolution of $18 \times 20$. And for the training of our detector, we used 9996 images of faces. For the test phase, we used the standard MIT + CMU base which contains 117 images with 511 faces.

The result of our work is the creation of two detectors UMIST-Detector and PIE-Detector from the process of learning using the two image bases, respectively, UMIST and PIE CMU described above.

The learning phase uses more than 300,000 features (or weak classifiers) divided into two types: normal and rotated. The storage of this large number of features took on a large amount of memory. The result of this phase is a detector composed of a group of features selected by the AdaBoost algorithm. For each feature selected, it takes 
about 3 to 4 hours - sometimes more - calculating time using an HP Notebook PC with a $2.6 \mathrm{GHz}$ frame rate and $4 \mathrm{~GB}$ of memory. This slowness of learning was considered a major drawback ofthe Viola \& Jones method.

Table 1:Number of normal and rotated Haar-Like features selected by AdaBoost for each DataBase.

\begin{tabular}{|c|c|c|c|}
\hline & UMIST & CMU-PIE & VIOLA \& JONES \\
\hline Normal & 4103 & 4075 & 4297 \\
\hline Rotated & 6424 & 4393 & 0 \\
\hline TOTAL & 10527 & 8468 & 4297 \\
\hline
\end{tabular}

Our program was run for 21 days for the first experience and 15 days for the second. The first detector consists of 10527 features distributed over 53 stages, and the second detector contains 8468 features spread over 46 stages.

The Viola-Jones detector contains 4297 features distributed over 32 stages, as is presented in the article of its authors [2][3]. The difference in the number of weak classifiers of our detectors and that of Viola and Jones is because we use, at most of the original features, those rotated by different angles. Table I shows the numbers of normal and rotated features selected by AdaBoost for each experiment, compared by the number obtained by Viola and Jones. Indeed, this added value has allowed us to achieve good results. These results clearly show that our detectors have correct detection rates greater than or nearly equal to those reported by Viola-Jones. UMISTDetector has a detection accuracy of up to $97.8 \%$, and this amounts to the fact that the images of the UMIST database are better pre-processed and standardized at the level of illumination, rotation of faces, different human races, etc. With PIE-Detector the detection rate cannot exceed the $95.9 \%$ threshold. To have performance tests comparable to those performed by Viola \& Jones and Rowly [2][3][11], we used the MIT-CMU test database, which consists of 117 images and 511 faces. Viola and Jones used 130 images and 507 faces. A difference that we neglected because most of the images were the same or have undergone some slight modifications. Figure. 8 illustrates these results as a ROC.

The results are generally better, especially those obtained by Viola and Jones. Our two detectors have lower false alarm rates than the other methods; $28 \%$ for the first experience and $31 \%$ for the second. By using a $2.3 \mathrm{GHz}$ core i3 and a 4GB memory capacity, our detectors can scan an image of $252 \times 426$ pixels in about 0.9 seconds with a scaling factor of 1.2. what makes thema little bitslow compared to that of Viola and Jones $(0.7$ seconds to scan an image of $384 \times 288$ ), and this is mainly due to the large number of weak classifiers used by our method. The average speed to determine all the key points for each rotated feature does not exceed $62.4 \mu \mathrm{s}$.

\section{CONCLUSION}

In this work, we have proposed a simple, and effective method to integrate a rectangle rotated by any angle of rotation. The principle of this technique is to findspecific pixels (key points) for each segment of the rectangle, then calculate the sum of its pixels according to these points. To find these points, we adopted the Bresenham algorithm for drawing a segment. This technique applied to the Viola and Jones algorithm with rotated HaarLike features added to the core set has provenefficiency and a high detection rate for detecting rotated faces in an image. In fact, we performed two experiments using, for the training phase, two image databases known in the literature, notably UMIST and CMU-PIE, and the MIT-CMU database for the test phase. Our perspective is to apply this technique for real-time detection and also for face and emotion recognition.

\section{REFERENCES}

1. Y. Freund et R. Schapire, "A desicion-theoretic generalization of on-line learning and an application to boosting", Journal of Computer and System Sciences(JCSS), vol. 55, no 1, 1997, p. 119-139 https://doi.org/10.1006/jcss.1997.1504

2. Viola P, Jones M. Robust real-time object detection. International Journal of Computer Vision (IJCV), 2001, 57(2), p. 137-154.

3. P. Viola, M. Jones. "Rapid Object Detection Using a Boosted Cascade of Simple Features", IEEE Computer Society Conference on Computer Vision and Pattern Recognition, Vol. 1, pp. 511-518, 2001.

4. P. Viola, M. Jones, D. Snow. "Detecting pedestrians using patterns of motion and appearance", International Journal of Computer Vision, Vol. 63, Issue 2, pp. 153-161, July 2005.

5. Lienhart R, Maydt J. "An extended set of haar-like features for rapid object detection", International Conference on. IEEE, 2002, vol. 1, pp: 900-903.

6. Miyamoto R, Sugano H, Saito H, et al. "Pedestrian recognition in far-infrared images by combining boosting-based detection and skeleton-based stochastic tracking", Advances in Image and Video Technology. Springer Berlin Heidelberg, 2006, pp: 483-494. https://doi.org/10.1007/11949534_48

7. A.L.C. Barczak. "Toward an Efficient Implementation of a Rotation Invariant Detector using Haar-Like Features", Proceedings of the IEEE/RSJ international conference on Intelligent robots and systems, Nouvelle-Zélande, Dunedin, 2005.

8. S. Du, N. Zheng, Q. You, Y. Wu, M. Yuan, J. Wu. "Rotated Haar-Like Features for Face Detection with In-Plane Rotation", 12th International Conference, VSMM 2006, Xi'an, China, October 18-20, Proceedings, pp 128-137, 2006.

9. C. H. Messom, A. L. C. Barczak, "Stream processing for fast and efficient rotated Haarlike features using rotated integral images". IJISTA 7(1): 40-57 (2009). https://doi.org/10.1504/IJISTA.2009.025105

10.G. A. Ramirez, O. Fuentes, "Multi-Pose Face Detection With Asymmetric Haar Features", 
Applications of Computer Vision, WACV, 2008, IEEE Workshop in Copper Mountain,CO.

11.H.A. Rowley, S. Baluja; T. Kanade, "Neural networkbased face detection", In IEEE Trans. Pattern Analysis and Machine Intelligence, vol.20 (1998), p. 23-38.

12. M. Oualla, A. Sadiq, S. Mbarki. "A new approach to represent rotated haar-like features for objects detection", Journal of Theoretical \& Applied Information Technology (JATIT), Vol.78. $\mathrm{N}^{\circ}$ 1, pp 15 - 23, 10th August 2015.

13.Jack E. Bresenham, "Algorithm for computer control of a digital plotter”, IBM Systems Journal, vol. 4, no 1, 1 th January 1965, p. 25-30.

14.M.T. Pham, Y. Gao, V.D.D. Hoang, T.J. Cham, "Fast Polygonal Integration and Its Application in Extending Haar-like Features to Improve Object Detection", In proceeding of the IEEE conference on Computer Vision and Pattern Recognition (CVPR); San Francisco, CA, 2010.

15.G. Doretto, T. Sebastian, P. Tu, and J.Rittscher. "Appearance-based person reidentification in camera networks: Problem overview and current approaches". In Journal of Ambient Intelligence and Humanized Computing, pp. 1-25, Springer Berlin / Heidelberg, 2011. https://doi.org/10.1007/s12652-010-0034-y

16. Crow, F. "Summed-area tables for texture mapping", SIGGRAPH, 84, pp. 207-212, 1984.

17.D.B. Graham and N.M. Allinson, "Characterizing Virtual Eigen signatures for General Purpose Face Recognition", NATO Advanced Study Institute, Face recognition: from theory to applications; 1997; Stirling in NATO ASI SERIES F COMPUTER AND SYSTEMS SCIENCES, vol. 163, pp. 446-456, (Springer), 1998.

18.T. Sim, S. Baker, M. Bsat. "The CMU Pose, Illumination, and Expression (PIE) Database of Human Faces", Transactions on Pattern Analysis and Machine Intelligence, Vol. 25(12): pp. 1615-1618, 2003.

19.A. D. M. Africa, G. Borja, A. Chua, D. Ong, M. Roque. "Application of Computer Systems in Facial Recognition Efficiency", International Journal of Advanced Trends in Computer Science and Engineering (IJATCSE), Vol. 8, No. 4, July-August 2019, https://doi.org/10.30534/ijatcse/2019/26842019

20. S. Chokkadi, S. MS, S. K B, A. Bhandary. "A Study on various state of the art of the Art Face Recognition Systemusing Deep Learning Techniques", International Journal of Advanced Trends in Computer Science and Engineering (IJATCSE), Vol. 8, No. 4, July - August 2019, https://doi.org/10.30534/ijatcse/2019/84842019. 